\title{
Antibiotic Susceptibility Patterns of Vibrio cholerae isolates
}

\author{
Shrestha SD, 1,2 Malla S, ${ }^{1}$ Adhikari BR, ${ }^{1}$ Shakya G, ${ }^{1}$ Basnyat SR, ${ }^{2}$ Sharma S' \\ 'National Public Health Laboratory, Teku, Kathmandu, ${ }^{2}$ Central Department of Microbiology, Tribhuvan University, Kirtipur, \\ Nepal.
}

\section{ABSTRACT}

Introduction: Cholera is one of the most common diarrhoeal diseases in Nepal. Etiological agent of cholera is Vibrio cholerae which removes essential body fluids, salts and vital nutrients, which are necessary for life causing dehydration and malnutrition. Emerging antimicrobial resistant is common. The aim of the present study was to determine the antibiotic susceptibility pattern of cholera patients in Nepal.

Methods: All the laboratory works were conducted in the bacteriology section of National Public Health Laboratory, Teku from March to September 2005. During this period a total of 340 stool samples from diarrhoeal patients were collected and processed according to the standard laboratory methods. Each patient suffering from diarrhoea was directly interviewed for his or her clinical history during sample collection.

Results: A total of 340 stool samples were processed and studied from both sex including all ages of patients. Among the processed sample 53 Vibrio cholerae cases were found. All isolated Vibrio cholerae O1 were El Tor, Inaba. All isolated (100\%) Vibrio cholerae O1 were sensitive to Ampicillin, Ciprofloxacin, Erythromycin and Tetracycline whereas all were resistant to Nalidixic acid and Cotrimoxazole. Only $15.1 \%$ cases were sensitive to Furazolidone whereas $84.9 \%$ were resistant.

Conclusion: All $V$. cholerae strains isolated in this study were found resistant to Multi Drug Resistant (resistant to at least two antibiotics of different group). Ampicillin, Ciprofloxacin, Erythromycin and Tetracycline were found still more potent antibiotics against Vibrio cholerae isolated during the study.

Keywords: antibiotics, susceptibility, Vibrio cholera

\section{INTRODUCTION}

Cholera is a disease caused by Vibrio cholerae. Since 1817, six pandemics have swept over the world, and the seventh one is in progress. ${ }^{1}$ Seventh pandemic was started from an Indonesian island which was caused by the E1 Tor biotype and it spread across Asia and
Africa. ${ }^{2}$ In Nepal, owing to the low socioeconomic status and poor hygienic condition of the people, Cholera constitute an important cause of morbidity and mortality. ${ }^{3}$ The case fatality rate in Nepal from diarrhoea is highest $(2 \%)$ in the age group of $1-4$ and is lowest (1\%) among the infants. ${ }^{4}$

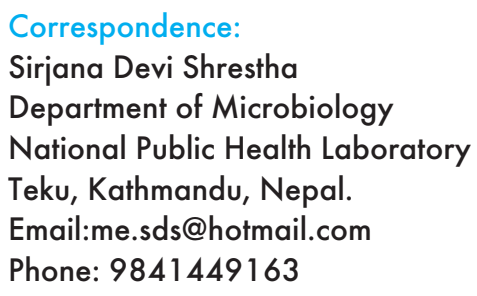


Shrestha et al. Antibiotic Susceptibility Patterns of Vibrio Cholerae isolates in Kathmandu Valley

There are several serogroups of $V$. cholera, the 01 serogroup being the cause of endemic and epidemic cholera. Using specific antisera to detect different fractions of the $O$ antigen, the 01 serogroup is divided into Inaba (AC), Ogawa (AB) and Hikojima (ABC) serotypes. ${ }^{5}$ Drug resistant $V$. cholera have been reported since 1977 and some of these strains were multidrug resistant. ${ }^{6,7}$ Due to the development of these resistant strains, it is now important to know the local antimicrobial susceptibility patterns of $V$. cholerae.

\section{METHODS}

This observational study was conducted in the bacteriology section of National Public Health Laboratory (NPHL), Teku, Kathmandu from March to September 2005, following standard microbiological techniques. Each patient suffering from diarrhoea was directly interviewed for his or her demographic profile (name, age, sex, address and hospital treated) and significant clinical history (sign, symptoms, stool passage frequency, stool consistency, presence of blood and mucus in the stool) during sample collection. Data was entered on a standard excel worksheet. Data analysis was performed by using Microsoft Excel 2007 and statistical package of social sciences (SPSS).

Stool sample was primarily inoculated on the plate of MacConkey Agar (MA) medium and Thiosulphate Citrate Bile Salt Sucrose (TCBS) medium, at the same time a loopful sample was inoculated in Alkaline Peptone Water (APW) for enrichment and incubated for 4-6 hours. From APW, sample was subcultured on second plate of MA and TCBS. All inoculated plates were incubated aerobically at $37^{\circ} \mathrm{C}$ for 24 hours. Vibrio suspected non-lactose fermenting colonies on MA and sucrose fermenting yellow colonies on TCBS were subcultured on Nutrient Agar (NA) medium and incubated for 24 hours at $37^{\circ} \mathrm{C}$. Further processing was performed from the colonies on NA plate.

Table 1. Age and gender wise distribution of samples and $\boldsymbol{V}$. cholera cases

\begin{tabular}{lcccc}
\hline \multirow{2}{*}{ Age groups } & \multicolumn{2}{c}{ Male } & \multicolumn{2}{c}{ Female } \\
& No. of patient & V. cholera cases & No. of patient & V. cholera cases \\
\hline $0-10 y r$ & 19 & 1 & 10 & 0 \\
$11-20 y r$ & 38 & 9 & 19 & 3 \\
$21-30 y r$ & 54 & 10 & 42 & 9 \\
$31-40 y r$ & 37 & 5 & 25 & 6 \\
$41-50 y r$ & 24 & 3 & 14 & 2 \\
$51-60 y r$ & 18 & 2 & 16 & 2 \\
$61-70 y r$ & 6 & 0 & 10 & 0 \\
$71-80 y r$ & 3 & 1 & 5 & 0 \\
$>80 y r$ & 0 & 0 & 0 & 0 \\
\hline Total no. (\%) & $199(58.52 \%)$ & $31(58.49 \%)$ & $141(41.47 \%)$ & $22(41.75 \%)$ \\
\hline
\end{tabular}

Identification was done by colony characteristics, Gram's stain, different biochemical tests (Different sugar utilization, Oxidase, Catalase, Indole, Methyl Red, VogesProskaur, Citrate Utilization, Oxidative-Fermentative, Motility, Urea Hydrolysis, Salt Concentration Tolerance and String test), serotyping ( $\mathrm{O}$-antigen groups were serologically identified by using commercially available polyvalent $\mathrm{O}$, polyvalent $\mathrm{O} 1$, monospecific Ogawa-Inaba antisera and with specific anti-O 139 antisera, Denka Seiken Co., Japan), and biotyping of Vibrio cholerae 01 (Haemagglutination of Chick RBC, Haemolysis of Sheep RBC, Susceptibility to Polymyxin B and VP Reaction).

Antibiotic susceptibility testing was done on MuellerHinton Agar plate by Kirby-Bauer disc diffusion method. Sensitivity of Ampicillin, Nalidixic acid, Ciprofloxacin, Cotrimoxazole, Erythromycin, Furazolidone, and Tetracycline were tested for Vibrio cholerae. The results were categorized as sensitive, resistant and partially sensitive based on standardized zones of inhibition.

\section{RESULTS}

Out of 340 patients, $82.94 \%$ were from Shukraraj Tropical Hospital and $17.05 \%$ were from different locality of Kathmandu valley attending NPHL by requesting stool culture. There were total of 53 $(15.58 \%)$ strains of $V$. cholerae and all of them were El Tor, Inaba. Association of isolation between male and female was not statistically significant. Majority of the patients were belonged to age group 21-30 years (Table 1). Isolation rate was found highest in August $(45.28 \%)$ (Figure 1). Vomiting, dehydration, nausea and passage of rice watery stool with more than 10 times per day were reported as the main clinical features of the cholera (Table $2 \& 3$ ). All isolates were sensitive to Ciprofloxacin, Ampicillin, Erythromycin and Tetracycline. Only $15.1 \%$ isolates were sensitive towards Furazolidon but all isolates were resistant to Nalidixic acid and Cotrimoxazole (Table 4). Hundred percent $V$. cholerae isolates were Multi-Drug resistant. 
Shrestha et al. Antibiotic Susceptibility Patterns of Vibrio Cholerae isolates in Kathmandu Valley

Table 2. Clinical symptoms exhibited by cholera patients

\begin{tabular}{cccccc}
\hline \multirow{2}{*}{ No. of V. cholerae isolates } & \multicolumn{2}{c}{ Symptoms } & & \\
& Abdominal pain/cramp & Fever & Vomiting & Dehydration & Nausea \\
\hline \multirow{2}{*}{53} & 6 & 2 & 51 & 47 & 31 \\
& $(11.3 \%)$ & $(3.77 \%)$ & $(96.2 \%)$ & $(88.69 \%)$ & $(58.4 \%)$ \\
\hline
\end{tabular}

Table 3. Physical characteristics of stool exhibited by cholera patients

\begin{tabular}{|c|c|c|c|c|c|c|}
\hline \multicolumn{2}{|c|}{$\begin{array}{c}\text { Stool passage frequency Per } \\
\text { day }(\%)\end{array}$} & \multicolumn{3}{|c|}{ Stool texture (Consistency) } & \multicolumn{2}{|c|}{$\begin{array}{c}\text { Presence of blood or mucus in } \\
\text { the stool }\end{array}$} \\
\hline$\geq 3$ & $\geq 10$ & Loose & Watery & $\begin{array}{c}\text { Rice } \\
\text { watery }\end{array}$ & Bloody & Mucoid \\
\hline $6(11.32 \%$ & $47(88.67 \%)$ & 0 & $13(24.52 \%)$ & $40(75.47 \%)$ & 0 & $11(20.7 \%)$ \\
\hline
\end{tabular}

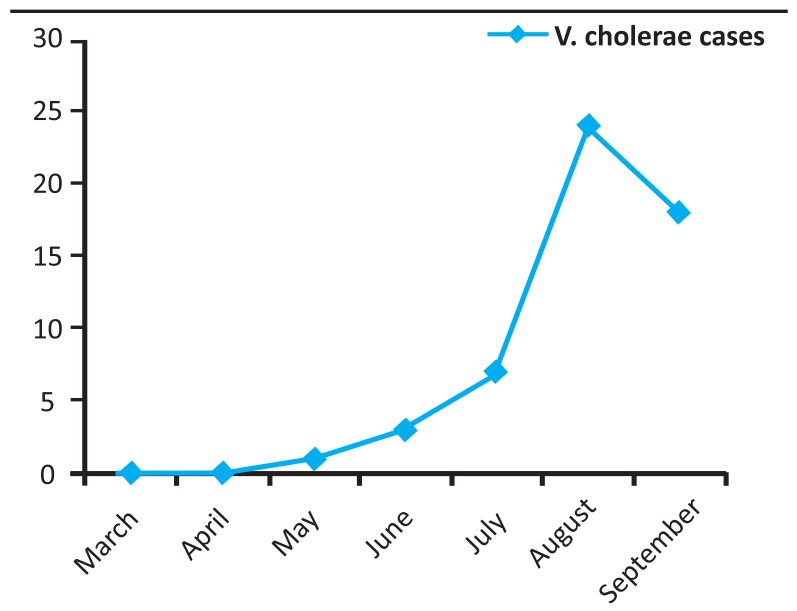

Figure 1. Seasonal distribution of V. cholerae

Table 4. Antibiotic susceptibility pattern of Vibrio cholerae $01(n=53)$

\begin{tabular}{lll}
\hline Antimicrobial & Sensitive & Resistant \\
Ampicillin & $53(100 \%)$ & 0 \\
Ciprofloxacin & $53(100 \%)$ & 0 \\
Tetracycline & $53(100 \%)$ & 0 \\
Erythromycin & $53(100 \%)$ & 0 \\
Furozolidone & $8(15.1 \%)$ & $45(84.9 \%)$ \\
Nalidixic Acid & 0 & $53(100 \%)$ \\
Cotrimoxazole & 0 & $53(100 \%)$ \\
\hline
\end{tabular}

\section{DISCUSSION}

Cholera is an important cause of acute diarrhoeal diseases in Nepal. Poor environmental hygiene, poor water sanitation and improper management of sewage wastes are the predominant factors that $V$. cholerae are established in Kathmandu valley. V. cholerae is one of the most important enteropathogens in all countries. ${ }^{8}$ The sixth pandemic of cholera and, presumably, the earlier pandemics were caused by the classical biotype of Vibrio cholerae 01, which was progressively replaced by the El Tor biotype representing the seventh cholera pandemic. ${ }^{9}$ Eighth pandemic in cholera history, could be due to Vibrio cholerae 01 El Tor, Inaba serotype, infection. ${ }^{10}$ Our result also support this view.

It was reported that for the last six years $V$. cholerae El Tor 01 Ogawa was responsible for the cholera endemic in Kathmandu, but in 1990 it was V. cholerae El Tor 01 Inaba, so it can be said that after 15 years later V. cholerae 01 Inaba emerged hugely in Kathmandu. ${ }^{11}$ Some previous outbreaks of cholera in Kathmandu valley like, in 1994 and in 2004 were due to $V$. cholerae, El Tor, 01 Ogawa. ${ }^{12,13}$ Although Ogawa was predominant, frequently Hikojima and to less often Inaba serotypes was also isolated in Nepal. ${ }^{14}$

Vibrio cholerae 01 strains with a periodic shift of different serotypes (Inaba and Ogawa) may be a consequence of genetic reversion which is known to take place in vitro and vivo. ${ }^{15,16}$ The only changes observed between the Ogawa and Inaba serotypes are related to a mutation in the $w b e T$ region on a 21.6 $\mathrm{kb}$ Sacl fragment of DNA. ${ }^{16,17}$ The epidemic strains had undergone a serotype conversion, possibly because of immune pressure in the population; or due to seletive pressure of anti-Ogawa antibodies and the emergence of anti-Inaba antibodies. ${ }^{15,18}$

In the present study, the highest cholera cases were seen among the age group between 21-30 years and were predominant among male patients. Although children are the primary victims of cholera, the present result may be due to higher frequency of adult patients visiting NPHL. The incidence of cholera are frequently reported in August and September. ${ }^{14,19}$ Vomiting, dehydration, nausea with rice watery stool and stool passage frequency $\geq 10$ were found the important clinical manifestations exhibited by cholera patients which are the universal clinical features of cholera.

Antibiotics shorten the course and diminish the severity of the illness, but they are not as important as rehydration, which is the cornerstone of therapy for cholera. The susceptibility pattern of the isolates, against seven different antibiotics, belonging to different groups were studied and found all with multiple antibiotic resistant capacity. It appears that antibiotic-resistant strains are 
Shrestha et al. Antibiotic Susceptibility Patterns of Vibrio Cholerae isolates in Kathmandu Valley

increasingly being found worldwide..$^{20,21}$

Since, Tetracycline is the drug of choice for cholera because it is more effective, non-toxic, easily available and comparatively cheap. In our study and in some other studies, all isolates were sensitive to Tetracycline and Cotrimoxazole. ${ }^{22}$ But Tetracycline, Cotrimoxazole and Furazolidon resistant $V$. cholerae strains were also reported. ${ }^{11,23}$ Resistance to Cotrimoxazole, Furazolidone and Streptomycin suggests the possibility of the presence of the SXT element in Inaba strains. ${ }^{24}$ Genes and their mechanisms of transfer may be important in the maintenance and transfer of resistance among V. cholerae and other enteric pathogens. In 1989, Erythromycin was reported to be effective for the treatment of Tetracycline resistant strains. ${ }^{25}$ All isolates in this study were found sensitive towards Erythromycin.

This study can help health authorities to review the cholera treatment guideline. Health education campaigns about prevention of diarrhoeal diseases and proper disposal of sewage, good sanitation and hygiene may be effective practice to control the cholera in Kathmadu Valley.

\section{CONCLUSIONS}

In Kathmandu Valley there is an incidence of cholera in the rainy season almost every year. The marked ability of $V$. cholera isolates to resist the inhibitory action of antibiotics has become a global problem. Increasing antibiotic resistance against commonly prescribed drugs signify that treatment options have become difficult in cases of cholera. The results indicate a continuing need for resistance surveillance and rational use of antimicrobial agents to reduce the multi-drug resistant strains of Vibrio.

\section{ACKNOWLEDGEMENT}

Authors would like to acknowledge all staff of NPHL for kind support and good co-operation during this study period.

\section{REFERENCES}

1. Rabbani HG, Greenough WB. Food as a Vehicle of Transmission of Cholera. Clinical Sciences Division, ICDDR, B: Centre for Health and Population Research. Journal of Diarrhoeal Diseases. 2002

2. Murray PR, Baron EJ. Manual of Clinical Microbiology. 9th ed. ASM Press; 2007

3. Sherchand JB, Larsson S, Shrestha MP. Intestinal parasites in children and adults with and without abdominal discomfort from the Kathmandu area of Nepal. Trop Gastroenterol. $1996 ; 17: 15-22$.

4. Bista MB, Shrestha K, Devkota UN. Gastroenteritis, Encephalits, Meningitis and Kalazar. An Epidmiology Division, Ministry of Health, Teku, Kathmandu. 1996;3-27.

5. Chatterjee BD, Braude AI, Davis CE. Infectious Disease and Medical Microbiology. 2nd ed. 1986.

6. Mhalu FS, Mmari PW, Ijumba J. Rapid emergence of EI Tor $V$. cholera resistant to antimicrobial agents during first six months of fourth cholera epidemic in Tanzania. Lancet. 1979 Feb 17;1(8112):345-7.

7. Siddique AK, Salam A, Islam MS, et al. Why treatment centers failed to prevent cholera deaths among Rwandan refugees in Goma, Zaire. Lancet. 1995;345:359-61.

8. Sunoto P. Diarrhoeal problems in Southeast Asia. Southest Asian J Trop Med Health. 1982;13(3):306-18.

9. Nair GB, Faruque SM, Bhuiyan NA, Kamruzzaman M, Siddique AK, Sack DA. New variants of Vibrio cholerae O1 biotype El Tor with attributes of the classical biotype from hospitalized patients with acute diarrhea in Bangladesh. J Clin Microbiol. 2002 Sep;40(9):3296-9.
10. Salim MV. Features of Vibrio cholerae 0:1 El Tor, Inaba serotype, isolated during the cholera epidemic in Cartagena (Colombia). Enferm Infecc Microbial Clin. 1992;10(9): 525-30.

11. Shrestha CD. Outbreak of cholera in Kathmandu Valley. J Nepal Med Assoc. 1997;35:66-9.

12. Ise T, Pokhrel BM, Rawal S, Shrestha RS. Outbreak of Cholera in the Kathmandu Valley, Nepal. J Inst Med. 1994;16:196-201.

13. Malla S, Kansakar P, Ghimire G. Cholera Outbreak in Kathmandu Valley in 2004: A review of national public health laboratory findings. Journal of Nepal Association for Medical Laboratory Science. 2005;7(7):20-3.

14. Pokhrel BM, Prasai BR, Kubo T, Sharma AP, Tuladhar NR, Shrestha SP, et al. Bacteriology of diarhoea in Nepal. J Inst Med. 1997;19:128-31.

15. Gangarosa EJ, Sanati A, Saghari H, Feeley JC. Multiple serotypes of Vibrio cholerae isolated from a case of cholera. Lancet. 1967;1:646-8.

16. Stroeher UH, Karageorgos LE, Morona R, Manning PA. Serotype conversion in Vibrio cholerae O1. Proc Natl Acad Sci USA. 1992;89:2566-70.

17. Yamasaki S, Garg S, Nair GB, Takeda Y. Distribution of Vibrio cholerae $\mathrm{O} 1$ antigen biosynthesis genes among O139 and other non-O1 serogroups of Vibrio cholerae. FEMS Microbiol Lett. 1999;179:115-21.

18. Manning PA, Stroeher UH, Morona R. Vibrio cholerae and cholera: molecular to global perspectives. Molecular basis for O-antigen biosynthesis in Vibrio cholerae O1: Ogawa-Inaba switching. Washington, DC: ASM Press; 1994. p. 77-94. 
19. Shrestha KD. Acute Diarrhoeal Cases in Nepal during Outbreak Season. An Epidemiological Review. J Nepal Med Assoc. 1995;33:219-31.

20. Kay BA, Bopp CA, Wells JG. Isolation and identification of Vibrio cholerae $\mathrm{O} 1$ from fecal specimens. In Vibrio cholerae and Cholera. Molecular to Global Perspectives. Washington, DC: ASM Press; 1994. p. 3-25.

21. Weber JT, Mintz ED, Canizares R, Semiglia A, GomezI, Sempértgui R. Epidemic cholera in Ecuador: multidrugresistance and transmission by water and seafood. Epidemiology and Infection. 1994;112:1-11.

22. Begue RE, Castellares G, Hayashi KE, Ruiz R, Oberst R. Diarrhoeal Disease in Peru after the introduction of Cholera. Amr J Trop Med. 1994;51:581-9.
23. Ramamurthy T, Pal A, Bhattacharya MK, Bhattacharya SK, Chowdhury AS, Takeda Y, et al. Serovar, biotype, phage type, toxigenicity \& antibiotic susceptibility patterns of Vibrio cholerae isolated during two consecutive cholera seasons (1989-90) in Calcutta. Indian J Med Res. 1992;95:125-9.

24. Waldor MK, Tschape H, Mekalanos JJ. A new type of conjugative transposon encodes resistance to sulfamethoxazole, trimethoprim, and streptomycin in Vibrio cholerae O139. J Bacteriol. 1996;178:4157-65.

25. Burans JP, Podgore J, Mansour MM. Comparative trial of erythromycin and sulphatrimethorprim in the treatment of tetracycline-resistant Vibrio cholera 01. Trans Roy Soc Trop Med Hyg. 1989;83:836-8. 